\title{
Consumer Brand Preference towards Mobile Phone: Effect of Mobile Phone Attributes on Purchase Decision
}

\author{
Parveen Kumari ${ }^{1}$, Satinder Kumar ${ }^{2}$ \\ ${ }^{I}$ (Research Scholar, Department of Management Studies/ Punjabi University, Patiala, India) \\ ${ }^{2}$ (Assistant Professor, Department of Management Studies/Punjabi University, Patiala, India)
}

\begin{abstract}
A consumer sets a frame of preferences in his/her mind to choose or purchase a product or service of same or different brands or producers. Keeping in view the frame of references the present paper is an attempt to study the attributes affecting the purchase decision of consumers towards the purchase of the Mobile Phones and to recommend the policies which may be adopted by the advertisers to enhance awareness among the buyers. The study used primary data collected from a sample of 84 consumers with the help of a questionnaire and found that consumer perceived that Technical Features are most considered attributes of mobile phone followed by Looks, Image \& Resource; Entertainment plays a significant role in taking the decision to purchase Mobile Phones. Hence, it may be recommended that the producers or marketers should made mobile phone with enhanced technical features that results in image building of buyers in the society.
\end{abstract}

Key words- attributes, brand, consumer preference, entertainment, technical feature.

\section{INTRODUCTION}

The mobile phones dominate most of modern human in every movement of life. Which Nowadays is becoming a part of basic needs of a person as means of communication across the world during the last fifteen years. Every individual use mobile for not only communication purpose, but also it became a personal assistance to make an everyday life easier. The development of mobile communication technology e. g. wireless internet, mobile phone, MP3 player, GPS navigation system has been a long journey of innovation, which is constantly evolving and updating as a result of consumers changing needs and preferences (Mokhlis, S. \& Yaakop, A.Y. [1]).There is a problem of hard competition among various brands of mobile phones in the market. Every day a new phone is launched with enhanced features and technology. Nevertheless of the fact that this competition has provided the consumer a wide variety of mobile phones to choose from at the same time there is also confusion prevailing in the minds of consumer about which of particular mobile phone suits their requirements the most. This problem of choice is known as brand preference. Brand preference has negatively impacted the marketers as well as consumers. For marketers increase in competition leads to decrease in sales for mobile phones as market share is now divided among more sellers. For consumers, it involves opportunity cost, i.e. cost of foregoing best alternate. So the study of brand preference is of great importance for the marketers as well as consumers. So the marketers have to study the various mobile phone attributes that influence the consumer brand preference towards mobile phones to face this competition. Perhaps a study which investigates the Consumer Brand Preference towards Mobile Phones by Factor Analysis method could remedy the situation.

\subsection{Objectives}

To identify the attributes considered by the consumer while buying a mobile phone.

\section{REVIEW OF LITERATURE}

(Macro Analysis \& Consumer Research Organisation, 2004.[2]) conducted a study to investigate the attitude, usage patterns, the factors that trigger for purchase and awareness with regards to the recent launches of mobile handsets in the market. The finding shows that a substantial amount has adequate exposure 
to technology prior to their actual purchase, there was high awareness about 'new model launches' by various companies like Nokia, Motorola, Samsung and LG and majority of the respondent perceive a cell phone as the technology that offers convenience and make their life easier. (Chowdhury $\&$ Rahman, 2013.[3]) studied the relationship between demographic variables on the brand preference and identified the attributes that affect the choice behavior of mobile handsets as well as why the young consumers give special emphasis to some particular factors in the Chittagong metropolitan city. young respondents emphasize less importance on durability, price, others advice and opinion because of their swift switching attitude. As a whole, Samsung is competing with industry leader Nokia, Symphony, Sony Erricson and other brands grab a share of the youth market.

(Gopal \& Anjali \& Aakanksha, 2013.[4]) studied showed that in today's market medium screen phone is quite popular, followed by large screen phones while the only minority of buyers go for the small screen models as evident from the survey conducted on consumers. As far as the size and weight of handsets are considered, slim handsets are leading the consumer market, followed by medium and as for the weight, light weighted phones are more popular while heavy weighted are the least while medium weighted phones still hold a ground in the market as observed from the study conducted.

(Marumbwa and Thakur, 2013.[5]) conducted a study to identify brand image positively influence the consumer brand preference and increase in customer satisfaction levels would yield positive consumer brand preferences. (Rijal, 2013.[6]) studied the criteria that student preferred on brand while purchasing a mobile phone. In a study technical criteria and economic criteria play a very important role to prefer brand and internet and friends are the main two sources from where they get information about the branded mobile phones. Das (as cited in Sata, 2013.[7]) conducted an empirical research based on survey method. According to the study, a handset of reputed brand, smart appearance, and with advanced value added features, pleasure ability and usability; is the choice of young consumers; females in gender-group, post-graduates in level of educationgroup, students in occupational group, urban residents in the geographical area group plays most prominent role in buying decision of a mobile handset.

(Singh, 2012.[8]) conducted a study to identify the customer preferences towards various mobile phone handsets in Punjab. In order to cope up with the research purpose, primary data was collected and analyzed which showed that Price Consciousness, Multimedia options, Features and Looks and Brand Image were the four factors significant in influencing the purchase behavior of mobile phones. (Liao, Yu-Jui, 2012[9]) investigated the determinants in smart phone purchases and identified the market segmentation of smart phone. The result of the study shows that when buying a smart phone, a consumer who considers that product design and integration of hardware and software are important and that the convenience of transferring files or media display are not important would choose Apple's iPhone.

\section{RESEARCH METHODOLGY}

The research design used in this study is exploratory, descriptive, pure and empirical in nature. The present research paper attempts to identify the effect of mobile phone attributes on consumer brand preference. To achieve the said objective, only 29 question item of the questionnaire was used. The study used primary Data collected with the help of a well- structured questionnaire.

The sampling technique employed in research was a non probability sample method and judgment sampling technique was selected for the study of population. A total 100 questionnaire had been sent out and to which 84 respondents are received are included and time of data collected is October, 2014 to November, 2014.Further, to analyze and interpret the data frequency factor analysis were used for confirmatory data analysis. 


\section{Figures AND TABLES}

TABLE I: KMO and Bartlett's Test

\begin{tabular}{|l|l|l|}
\hline \multicolumn{2}{|l|}{ Kaiser-Meyer-Olkin Measure of Sampling Adequacy } & .780 \\
\hline \multirow{5}{*}{} & Approx. Chi-Square & 1.279 \\
\cline { 2 - 3 } & Df & 406 \\
\cline { 2 - 3 } & Sig. & .000 \\
\hline
\end{tabular}

Source: Primary data, (Processed through SPSS 16.0)

Table I shows the appropriateness of factor analysis technique the correlation between the variables is checked and Keiser-Meyer-Olkin (KMO) measure of sample adequacy is also used for the same. The test statistics for sphericity is based on Chi-square transformation of the determinants of the correlation matrix. Further, KMO compares the magnitude of the observed correlation coefficient to the magnitude of the partial correlation. The Small value of KMO indicates that the correlation between pairs of variables cannot be explained by other variables and factor analysis may not be appropriate. Generally a value greater than .5 is desired for the test statistic. It can be seen from the table 2 that the population correlation matrix is an identity matrix, is rejected from by Bartlett's Test of Sphericity. The approximate Chi-square value is 1.279 with 406 degree of freedom, which is significant at 0.05 level of significant. The value of KMO statistic, .780 is also larger than 0.5 . Thus, factor analysis may be considered as appropriate technique for analyzing the correlation matrix. The two basic approaches are principal component analysis (PCA) and common factor analysis (CFA). In PCA, total variance in the data is considered. PCA is recommended when the primary concern is to determine the minimum numbers of factors that will account for maximum variance in the data. Further, PCA may be carried out if correlation for variables contains at least two correlation of 0.30 or greater as said by F. Andy and M. Jeremy (as cited in Singh, 2013). The table 1 correlation matrix of 29 variables which were develops to know the opinion of consumer about the influence of mobile phones attributes on purchase decision and it is found that there is high correlation between variables; therefore it may be stated that factor analysis is appropriate. Therefore, PCA method is used for extraction of variables for the components and factor concerned.

TABLE II: Communalities

\begin{tabular}{|l|l|l|}
\hline & Initial & Extraction \\
\hline Camera & 1.000 & .763 \\
\hline Bluetooth & 1.000 & .733 \\
\hline MP 3 player & 1.000 & .771 \\
\hline Dual Sim & 1.000 & .662 \\
\hline Wi-Fi & 1.000 & .703 \\
\hline FM-Radio & 1.000 & .784 \\
\hline Brand image & 1.000 & .651 \\
\hline Shape & 1.000 & .685 \\
\hline Size of mobile phone & 1.000 & .676 \\
\hline Weight & 1.000 & .838 \\
\hline Colour & 1.000 & .793 \\
\hline Video-recorder & 1.000 & .757 \\
\hline Touch Screen & 1.000 & .725 \\
\hline Internet/GPRS & 1.000 & .746 \\
\hline Huge Memory & 1.000 & .756 \\
\hline Screen size & 1.000 & .641 \\
\hline
\end{tabular}


IOSR Journal of Business and Management (IOSR-JBM)

e-ISSN: 2278-487X, p-ISSN: 2319-7668

\begin{tabular}{|l|l|l|}
\hline Social Media Application & 1.000 & .704 \\
\hline Key Pad & 1.000 & .787 \\
\hline Communication & 1.000 & .619 \\
\hline Sending and receiving e-mail & 1.000 & .811 \\
\hline As a modem & 1.000 & .756 \\
\hline Playing games & 1.000 & .731 \\
\hline Strong battery back-up & 1.000 & .672 \\
\hline Design \& Appearance & 1.000 & .803 \\
\hline Sophisticated & 1.000 & .689 \\
\hline Good audio-video quality & 1.000 & .682 \\
\hline Operating System & 1.000 & .773 \\
\hline Video-calling facility & 1.000 & .804 \\
\hline Multi-Window & 1.000 & .776 \\
\hline Source: Primar Data (Procesed
\end{tabular}

Source: Primary Data (Processed through SPSS 16.0)

Extraction Method: Principal Component Analysis.

Table II shows that the extraction communalities for each variable which the amount of variance a variable share with all the other variables being considered. It is also the proportion of variance explained by the common factors.

TABLE III: Total Variance Explained

\begin{tabular}{|c|c|c|c|c|c|c|c|c|c|}
\hline \multirow[t]{2}{*}{ Component } & \multicolumn{3}{|c|}{ Initial Eigen values } & \multicolumn{3}{|c|}{\begin{tabular}{|l|}
$\begin{array}{l}\text { Extraction } \\
\text { Loadings }\end{array}$ \\
\end{tabular}} & \multicolumn{3}{|c|}{\begin{tabular}{|l}
$\begin{array}{l}\text { Rotation Sums of Squared } \\
\text { Loadings }\end{array}$ \\
\end{tabular}} \\
\hline & Total & $\begin{array}{l}\% \text { of } \\
\text { Variance }\end{array}$ & $\begin{array}{l}\text { Cumulative } \\
\%\end{array}$ & Total & $\begin{array}{l}\% \text { of } \\
\text { Variance }\end{array}$ & $\begin{array}{l}\text { Cumulative } \\
\%\end{array}$ & Total & $\begin{array}{l}\% \text { of } \\
\text { Variance }\end{array}$ & $\begin{array}{l}\text { Cumulative } \\
\%\end{array}$ \\
\hline 1 & 9.419 & 32.479 & 32.479 & 9.419 & 32.479 & 32.479 & 4.288 & 14.788 & 14.788 \\
\hline 2 & 2.076 & 7.159 & 39.638 & 2.076 & 7.159 & 39.638 & 3.186 & 10.986 & 25.774 \\
\hline 3 & 1.979 & 6.822 & 46.460 & 1.979 & 6.822 & 46.460 & 2.658 & 9.165 & 34.939 \\
\hline 4 & 1.818 & 6.268 & 52.728 & 1.818 & 6.268 & 52.728 & 2.464 & 8.496 & 43.435 \\
\hline 5 & 1.457 & 5.024 & 57.752 & 1.457 & 5.024 & 57.752 & 1.981 & 6.831 & 50.266 \\
\hline 6 & 1.233 & 4.253 & 62.005 & 1.233 & 4.253 & 62.005 & 1.898 & 6.544 & 56.810 \\
\hline 7 & 1.201 & 4.141 & 66.146 & 1.201 & 4.141 & 66.146 & 1.827 & 6.301 & 63.111 \\
\hline 8 & 1.082 & 3.730 & 69.876 & 1.082 & 3.730 & 69.876 & 1.780 & 6.138 & 69.249 \\
\hline 9 & 1.026 & 3.539 & 73.415 & 1.026 & 3.539 & 73.415 & 1.208 & 4.166 & 73.415 \\
\hline 10 & .879 & 3.032 & 76.446 & & & & & & \\
\hline 11 & .732 & 2.524 & 78.971 & & & & & & \\
\hline 12 & .701 & 2.418 & 81.389 & & & & & & \\
\hline 13 & .592 & 2.040 & 83.430 & & & & & & \\
\hline 14 & .570 & 1.964 & 85.394 & & & & & & \\
\hline 15 & .494 & 1.703 & 87.096 & & & & & & \\
\hline 16 & .477 & 1.646 & 88.743 & & & & & & \\
\hline 17 & .445 & 1.535 & 90.278 & & & & & & \\
\hline 18 & .386 & 1.333 & 91.610 & & & & & & \\
\hline
\end{tabular}


IOSR Journal of Business and Management (IOSR-JBM)

e-ISSN: 2278-487X, p-ISSN: 2319-7668

\begin{tabular}{|l|c|c|c|l|l|l|l|l|l|}
\hline 19 & .377 & 1.300 & 92.911 & & & & & & \\
\hline 20 & .344 & 1.185 & 94.096 & & & & & & \\
\hline 21 & .325 & 1.122 & 95.217 & & & & & & \\
\hline 22 & .277 & .955 & 96.172 & & & & & & \\
\hline 23 & .223 & .767 & 96.940 & & & & & & \\
\hline 24 & .208 & .719 & 97.658 & & & & & & \\
\hline 25 & .175 & .604 & 98.262 & & & & & & \\
\hline 26 & .165 & .568 & 98.831 & & & & & & \\
\hline 27 & .148 & .510 & 99.340 & & & & & & \\
\hline 28 & .101 & .349 & 99.689 & & & & & & \\
\hline 29 & .090 & .311 & 100.000 & & & & & & \\
\hline
\end{tabular}

Extraction Method: Principal Component Analysis.

Source: Primary (Data processed through SPSS 16.0)

Table III demonstrates that nine factors have been extracted on the basis of prior knowledge to describe the relationship among variable in a best way. Finally, from the commutative percentage of variance accounted for, 73.415 percent of the nine variables, contributed by first component is (32.479) followed by second (7.159), third (6.822), fourth (6.268), fifth (5.024), sixth (4.253), seventh (4.141), eight (3.730), and ninth (3.539) of total variance.

TABLE IV: Component (Factor) Pattern Matrix

\begin{tabular}{|c|c|c|c|c|c|c|c|c|c|c|c|c|c|c|c|c|c|c|}
\hline & \multicolumn{9}{|c|}{ Component } & \multicolumn{9}{|c|}{ Rotated Component Matrix } \\
\hline & 1 & 2 & 3 & 4 & 5 & 6 & 7 & 8 & 9 & 1 & 2 & 3 & 4 & 5 & 6 & 7 & 8 & 9 \\
\hline Camera & $\begin{array}{l}0 . \\
45 \\
8\end{array}$ & $\begin{array}{l}- \\
0 . \\
43\end{array}$ & $\begin{array}{l}0 . \\
12 \\
8\end{array}$ & $\begin{array}{l}- \\
0 . \\
37\end{array}$ & $\begin{array}{l} \\
0 . \\
35\end{array}$ & $\begin{array}{l}0 . \\
3\end{array}$ & $\begin{array}{l}- \\
0 . \\
06\end{array}$ & $\begin{array}{l}- \\
0 . \\
08\end{array}$ & $\begin{array}{l}0 . \\
05\end{array}$ & $\begin{array}{l}0 . \\
22 \\
3\end{array}$ & $\begin{array}{l}0 . \\
12 \\
5\end{array}$ & $\begin{array}{l}0 . \\
49\end{array}$ & $\begin{array}{l}0 . \\
18 \\
4\end{array}$ & $\begin{array}{l}- \\
0 . \\
06\end{array}$ & $\begin{array}{l}- \\
0 . \\
05 \\
6\end{array}$ & $\begin{array}{l}0 . \\
64 \\
5\end{array}$ & $\begin{array}{l}0 . \\
01 \\
9\end{array}$ & $\begin{array}{l}0 . \\
03 \\
7\end{array}$ \\
\hline $\begin{array}{l}\text { Bluetoot } \\
\mathrm{h}\end{array}$ & $\begin{array}{l}0 . \\
24 \\
7\end{array}$ & $\begin{array}{l}0 . \\
47 \\
7\end{array}$ & $\begin{array}{l}0 . \\
55 \\
3\end{array}$ & $\begin{array}{l}- \\
0 . \\
14\end{array}$ & $\begin{array}{l}0 . \\
16 \\
2\end{array}$ & $\begin{array}{l}0 . \\
0 \\
5\end{array}$ & $\begin{array}{l}0 . \\
29 \\
7\end{array}$ & $\begin{array}{l}0 . \\
02 \\
6\end{array}$ & $\begin{array}{l}0 . \\
04 \\
7\end{array}$ & $\begin{array}{l}0 . \\
05 \\
6\end{array}$ & $\begin{array}{l} \\
0 . \\
12\end{array}$ & $\begin{array}{l}- \\
0 . \\
02 \\
4\end{array}$ & $\begin{array}{l}0 . \\
79\end{array}$ & $\begin{array}{l}0 . \\
10 \\
2\end{array}$ & $\begin{array}{l}0 . \\
05 \\
4\end{array}$ & $\begin{array}{l} \\
0 . \\
23\end{array}$ & $\begin{array}{l}0 . \\
06 \\
9\end{array}$ & $\begin{array}{l}0 . \\
14 \\
2\end{array}$ \\
\hline $\begin{array}{ll}\text { MP } 3 \\
\text { player }\end{array}$ & $\begin{array}{l}0 . \\
53 \\
7\end{array}$ & $\begin{array}{l}0 . \\
17 \\
8\end{array}$ & $\begin{array}{l}0 . \\
52 \\
7\end{array}$ & $\begin{array}{l}- \\
0 . \\
24\end{array}$ & $\begin{array}{l}0 . \\
07 \\
5\end{array}$ & $\begin{array}{l}- \\
0 . \\
1\end{array}$ & $\begin{array}{l}0 . \\
13 \\
9\end{array}$ & $\begin{array}{l}- \\
0 . \\
13\end{array}$ & $\begin{array}{l}- \\
0 . \\
26\end{array}$ & $\begin{array}{l}0 . \\
13\end{array}$ & $\begin{array}{l}0 . \\
27 \\
3\end{array}$ & $\begin{array}{l}0 . \\
01 \\
3\end{array}$ & $\begin{array}{l}0 . \\
76 \\
4\end{array}$ & $\begin{array}{l}0 . \\
03 \\
4\end{array}$ & $\begin{array}{l}0 . \\
17 \\
4\end{array}$ & $\begin{array}{l}0 . \\
08 \\
4\end{array}$ & $\begin{array}{l}0 . \\
21 \\
8\end{array}$ & $\begin{array}{l}- \\
0 . \\
09 \\
1\end{array}$ \\
\hline $\begin{array}{l}\text { Dual } \\
\text { Sim }\end{array}$ & $\begin{array}{l}0 . \\
50 \\
1 \\
\end{array}$ & $\begin{array}{l}- \\
0 . \\
08\end{array}$ & $\begin{array}{l}0 . \\
21 \\
8 \\
\end{array}$ & $\begin{array}{l}0 . \\
42 \\
2\end{array}$ & $\begin{array}{l}- \\
0 . \\
26\end{array}$ & $\begin{array}{l}0 . \\
1 \\
2\end{array}$ & $\begin{array}{l}0 . \\
15 \\
7 \\
\end{array}$ & $\begin{array}{l}0 . \\
18\end{array}$ & $\begin{array}{l}0 . \\
19 \\
5\end{array}$ & $\begin{array}{l}0 . \\
11 \\
3\end{array}$ & $\begin{array}{l}0 . \\
26 \\
6 \\
\end{array}$ & $\begin{array}{l}0 . \\
60 \\
4 \\
\end{array}$ & $\begin{array}{l}0 . \\
15 \\
3 \\
\end{array}$ & $\begin{array}{l}- \\
0 . \\
03\end{array}$ & $\begin{array}{l}0 . \\
18 \\
3 \\
\end{array}$ & $\begin{array}{l}- \\
0 . \\
18\end{array}$ & $\begin{array}{l}- \\
0 . \\
04\end{array}$ & $\begin{array}{l}0 . \\
35\end{array}$ \\
\hline Wi-Fi & $\begin{array}{l}0 . \\
63 \\
1\end{array}$ & $\begin{array}{l} \\
0 . \\
27\end{array}$ & $\begin{array}{l}0 . \\
17 \\
2\end{array}$ & $\begin{array}{l}0 . \\
35 \\
7\end{array}$ & $\begin{array}{l} \\
0 . \\
1\end{array}$ & $\begin{array}{l}0 . \\
1 \\
1\end{array}$ & $\begin{array}{l}- \\
0 . \\
15\end{array}$ & $\begin{array}{l}- \\
0 . \\
02\end{array}$ & $\begin{array}{l}0 . \\
16 \\
3\end{array}$ & $\begin{array}{l}0 . \\
11 \\
3\end{array}$ & $\begin{array}{l}0 . \\
41 \\
6\end{array}$ & $\begin{array}{l}0 . \\
66 \\
4\end{array}$ & $\begin{array}{l}0 . \\
04 \\
1\end{array}$ & $\begin{array}{l}0 . \\
10 \\
8\end{array}$ & $\begin{array}{l}0 . \\
05 \\
3\end{array}$ & $\begin{array}{l}- \\
0 . \\
03\end{array}$ & $\begin{array}{l}0 . \\
22 \\
1\end{array}$ & $\begin{array}{l}0 . \\
10 \\
5\end{array}$ \\
\hline $\begin{array}{l}\text { FM- } \\
\text { Radio }\end{array}$ & $\begin{array}{l}0 . \\
11 \\
4\end{array}$ & $\begin{array}{l}0 . \\
49 \\
1\end{array}$ & $\begin{array}{l}0 . \\
15 \\
9\end{array}$ & $\begin{array}{l}0 . \\
56 \\
1\end{array}$ & $\begin{array}{l}0 . \\
07 \\
1\end{array}$ & $\begin{array}{l}0 . \\
0 \\
2\end{array}$ & $\begin{array}{l}0 . \\
27 \\
5\end{array}$ & $\begin{array}{l}- \\
0 . \\
25\end{array}$ & $\begin{array}{l}0 . \\
21 \\
5\end{array}$ & $\begin{array}{l}0 . \\
14 \\
8\end{array}$ & $\begin{array}{l}0 . \\
00 \\
1\end{array}$ & $\begin{array}{l}0 . \\
17 \\
8\end{array}$ & $\begin{array}{l}0 . \\
24 \\
1\end{array}$ & $\begin{array}{l} \\
0 . \\
1\end{array}$ & $\begin{array}{l}- \\
0 . \\
06 \\
8\end{array}$ & $\begin{array}{l}- \\
0 . \\
81\end{array}$ & -0 & $\begin{array}{l}0 . \\
05 \\
4\end{array}$ \\
\hline
\end{tabular}




\begin{tabular}{|c|c|c|c|c|c|c|c|c|c|c|c|c|c|c|c|c|c|c|}
\hline $\begin{array}{l}\text { Brand } \\
\text { image }\end{array}$ & $\begin{array}{l}0 . \\
58 \\
7\end{array}$ & $\begin{array}{l}- \\
0 . \\
16\end{array}$ & $\begin{array}{l}- \\
0 . \\
01\end{array}$ & $\begin{array}{l}0 . \\
10 \\
1\end{array}$ & $\begin{array}{l}- \\
0 . \\
32\end{array}$ & $\begin{array}{l}0 . \\
0 \\
4\end{array}$ & $\begin{array}{l} \\
0 . \\
26\end{array}$ & $\begin{array}{l}- \\
0 . \\
17\end{array}$ & $\begin{array}{l}0 . \\
26 \\
4\end{array}$ & $\begin{array}{l}0 . \\
27 \\
8\end{array}$ & $\begin{array}{l}0 . \\
16 \\
8\end{array}$ & $\begin{array}{l}0 . \\
67 \\
4\end{array}$ & $\begin{array}{l}- \\
0 . \\
04\end{array}$ & $\begin{array}{l}0 . \\
08\end{array}$ & $\begin{array}{l}0 . \\
14 \\
5\end{array}$ & $\begin{array}{l}0 . \\
09 \\
4\end{array}$ & $\begin{array}{l}0 . \\
13 \\
6\end{array}$ & $\begin{array}{l}- \\
0 . \\
18 \\
6\end{array}$ \\
\hline Shape & $\begin{array}{l}0 . \\
66\end{array}$ & $\begin{array}{l}- \\
0 . \\
33\end{array}$ & $\begin{array}{l}- \\
0 . \\
02\end{array}$ & $\begin{array}{l}0 . \\
14\end{array}$ & $\begin{array}{l}0 . \\
24\end{array}$ & $\begin{array}{l} \\
0 . \\
2\end{array}$ & $\begin{array}{l}0 . \\
07 \\
7\end{array}$ & $\begin{array}{l}- \\
0 . \\
07\end{array}$ & $\begin{array}{l}- \\
0 . \\
02\end{array}$ & $\begin{array}{l}0 . \\
21 \\
9\end{array}$ & $\begin{array}{l}0 . \\
67 \\
8\end{array}$ & $\begin{array}{l}0 . \\
20 \\
5\end{array}$ & $\begin{array}{l}0 . \\
08 \\
1\end{array}$ & $\begin{array}{l}0 . \\
35 \\
1\end{array}$ & $\begin{array}{l}0 . \\
03 \\
9\end{array}$ & $\begin{array}{l}0 . \\
01 \\
8\end{array}$ & $\begin{array}{l}0 . \\
05\end{array}$ & $\begin{array}{l}- \\
0 . \\
04 \\
1\end{array}$ \\
\hline $\begin{array}{l}\text { Size of } \\
\text { mobile } \\
\text { phone }\end{array}$ & $\begin{array}{l}0 . \\
59 \\
1\end{array}$ & $\begin{array}{l}- \\
0 . \\
41\end{array}$ & $\begin{array}{l}- \\
0 . \\
03\end{array}$ & $\begin{array}{l}0 . \\
26 \\
1\end{array}$ & $\begin{array}{l}0 . \\
19\end{array}$ & $\begin{array}{l}0 . \\
0 \\
3\end{array}$ & $\begin{array}{l}0 . \\
04 \\
5\end{array}$ & $\begin{array}{l}- \\
0 . \\
09\end{array}$ & $\begin{array}{l}- \\
0 . \\
2\end{array}$ & $\begin{array}{l}0 . \\
20 \\
6\end{array}$ & $\begin{array}{l}0 . \\
72\end{array}$ & $\begin{array}{l}0 . \\
21 \\
4\end{array}$ & $\begin{array}{l}- \\
0 . \\
01\end{array}$ & $\begin{array}{l}0 . \\
10 \\
6\end{array}$ & $\begin{array}{l}- \\
0 . \\
08 \\
6\end{array}$ & $\begin{array}{l}0 . \\
07\end{array}$ & $\begin{array}{l}0 . \\
18 \\
9\end{array}$ & $\begin{array}{l}0 . \\
09 \\
5\end{array}$ \\
\hline Weight & $\begin{array}{l}0 . \\
1\end{array}$ & $\begin{array}{l}- \\
0 . \\
01\end{array}$ & $\begin{array}{l}0 . \\
03 \\
5 \\
\end{array}$ & $\begin{array}{l}0 . \\
3\end{array}$ & $\begin{array}{l}0 . \\
13 \\
7 \\
\end{array}$ & $\begin{array}{l}0 . \\
4 \\
6 \\
\end{array}$ & $\begin{array}{l}0 . \\
21 \\
2 \\
\end{array}$ & $\begin{array}{l}0 . \\
67 \\
4 \\
\end{array}$ & $\begin{array}{l}- \\
0 . \\
1\end{array}$ & $\begin{array}{l}0 . \\
00 \\
2 \\
\end{array}$ & $\begin{array}{l}0 . \\
05 \\
3 \\
\end{array}$ & $\begin{array}{l}0 . \\
01 \\
6 \\
\end{array}$ & $\begin{array}{l}- \\
0 . \\
02\end{array}$ & $\begin{array}{l}0 . \\
01 \\
5 \\
\end{array}$ & $\begin{array}{l}0 . \\
00 \\
3\end{array}$ & $\begin{array}{l}- \\
0 . \\
03\end{array}$ & $\begin{array}{l}0 . \\
10 \\
4 \\
\end{array}$ & $\begin{array}{l}0 . \\
90 \\
7 \\
\end{array}$ \\
\hline Colour & $\begin{array}{l}0 . \\
47 \\
2\end{array}$ & $\begin{array}{l}- \\
0 . \\
34\end{array}$ & $\begin{array}{l}- \\
0 . \\
01\end{array}$ & $\begin{array}{l}0 . \\
44 \\
8\end{array}$ & $\begin{array}{l}- \\
0 . \\
06\end{array}$ & $\begin{array}{l}- \\
0 . \\
5\end{array}$ & $\begin{array}{l}0 . \\
07 \\
9\end{array}$ & $\begin{array}{l}0 . \\
16 \\
1\end{array}$ & $\begin{array}{l}- \\
0 . \\
09\end{array}$ & $\begin{array}{l}- \\
0 . \\
03 \\
7\end{array}$ & $\begin{array}{l}0 . \\
72\end{array}$ & $\begin{array}{l}0 . \\
24 \\
7\end{array}$ & $\begin{array}{l}- \\
0 . \\
12\end{array}$ & $\begin{array}{l}0 . \\
13 \\
6\end{array}$ & $\begin{array}{l}0 . \\
38 \\
2\end{array}$ & $\begin{array}{l} \\
0 . \\
13\end{array}$ & $\begin{array}{l}- \\
0 . \\
11\end{array}$ & $\begin{array}{l}0 . \\
06 \\
9\end{array}$ \\
\hline $\begin{array}{l}\text { Video- } \\
\text { recorder }\end{array}$ & $\begin{array}{l}0 . \\
51 \\
4\end{array}$ & $\begin{array}{l}0 . \\
24 \\
1\end{array}$ & $\begin{array}{l}0 . \\
22\end{array}$ & $\begin{array}{l}- \\
0 . \\
12\end{array}$ & $\begin{array}{l}- \\
0 . \\
43\end{array}$ & $\begin{array}{l}- \\
0 . \\
2\end{array}$ & $\begin{array}{l}- \\
0 . \\
05\end{array}$ & $\begin{array}{l}0 . \\
33 \\
7\end{array}$ & $\begin{array}{l}- \\
0 . \\
11\end{array}$ & $\begin{array}{l}0 . \\
15 \\
1\end{array}$ & $\begin{array}{l}0 . \\
03 \\
8\end{array}$ & $\begin{array}{l}0 . \\
22 \\
7\end{array}$ & $\begin{array}{l}0 . \\
33 \\
2\end{array}$ & -0 & $\begin{array}{l}0 . \\
73 \\
7\end{array}$ & $\begin{array}{l}0 . \\
13 \\
5\end{array}$ & $\begin{array}{l}0 . \\
05 \\
5\end{array}$ & $\begin{array}{l}0 . \\
08 \\
5\end{array}$ \\
\hline $\begin{array}{l}\text { Touch } \\
\text { Screen }\end{array}$ & $\begin{array}{l}0 . \\
59 \\
6 \\
\end{array}$ & $\begin{array}{l}- \\
0 . \\
3\end{array}$ & $\begin{array}{l}0 . \\
14 \\
2 \\
\end{array}$ & $\begin{array}{l}- \\
0 . \\
12\end{array}$ & $\begin{array}{l}- \\
0 . \\
08\end{array}$ & $\begin{array}{l}- \\
0 . \\
1\end{array}$ & $\begin{array}{l}0 . \\
16\end{array}$ & $\begin{array}{l}- \\
0 . \\
08\end{array}$ & $\begin{array}{l}- \\
0 . \\
44\end{array}$ & $\begin{array}{l}0 . \\
22 \\
3 \\
\end{array}$ & $\begin{array}{l}0 . \\
64 \\
2 \\
\end{array}$ & $\begin{array}{l}0 . \\
04 \\
8 \\
\end{array}$ & $\begin{array}{l}0 . \\
31 \\
1 \\
\end{array}$ & $\begin{array}{l} \\
0 . \\
09\end{array}$ & $\begin{array}{l}0 . \\
19 \\
1 \\
\end{array}$ & $\begin{array}{l}0 . \\
33 \\
7\end{array}$ & $\begin{array}{l}0 . \\
07 \\
2 \\
\end{array}$ & $\begin{array}{l}- \\
0 . \\
04\end{array}$ \\
\hline $\begin{array}{l}\text { Internet/ } \\
\text { GPRS }\end{array}$ & $\begin{array}{l}0 . \\
67 \\
5\end{array}$ & $\begin{array}{l}- \\
0 . \\
21\end{array}$ & $\begin{array}{l}0 . \\
07 \\
6\end{array}$ & $\begin{array}{l}- \\
0 . \\
27\end{array}$ & $\begin{array}{l}0 . \\
14 \\
6\end{array}$ & $\begin{array}{l}0 . \\
1 \\
3\end{array}$ & $\begin{array}{l}0 \\
36 \\
2\end{array}$ & $\begin{array}{l}0 . \\
03 \\
4\end{array}$ & $\begin{array}{l}0 . \\
01 \\
7\end{array}$ & $\begin{array}{l}0 . \\
43 \\
2\end{array}$ & $\begin{array}{l}0 \\
38 \\
1\end{array}$ & $\begin{array}{l}0 . \\
15 \\
5\end{array}$ & $\begin{array}{l}0 . \\
42 \\
4\end{array}$ & $\begin{array}{l}0 . \\
27 \\
7\end{array}$ & $\begin{array}{l}- \\
0 . \\
08 \\
2\end{array}$ & $\begin{array}{l}0 \\
29 \\
3\end{array}$ & $\begin{array}{l}- \\
0 . \\
09\end{array}$ & $\begin{array}{l}0 . \\
18 \\
2\end{array}$ \\
\hline $\begin{array}{l}\text { Huge } \\
\text { Memory }\end{array}$ & $\begin{array}{l}0 . \\
64\end{array}$ & $\begin{array}{l}0 . \\
01 \\
5\end{array}$ & $\begin{array}{l}- \\
0 . \\
38\end{array}$ & $\begin{array}{l}- \\
0 . \\
25\end{array}$ & $\begin{array}{l}- \\
0 . \\
03\end{array}$ & $\begin{array}{l}0 . \\
2 \\
1\end{array}$ & $\begin{array}{l}0 . \\
18 \\
1\end{array}$ & $\begin{array}{l}- \\
0 . \\
21\end{array}$ & $\begin{array}{l}- \\
0 . \\
13\end{array}$ & $\begin{array}{l}0 . \\
79 \\
4\end{array}$ & $\begin{array}{l}0 . \\
22 \\
7\end{array}$ & $\begin{array}{l}0 . \\
05 \\
4\end{array}$ & $\begin{array}{l}0 . \\
09\end{array}$ & $\begin{array}{l}0 . \\
03 \\
6\end{array}$ & $\begin{array}{l}- \\
0 . \\
03 \\
9\end{array}$ & $\begin{array}{l}0 . \\
23 \\
1\end{array}$ & $\begin{array}{l}0 . \\
06 \\
6\end{array}$ & $\begin{array}{l}- \\
0 . \\
04 \\
1\end{array}$ \\
\hline $\begin{array}{l}\text { Screen } \\
\text { size }\end{array}$ & $\begin{array}{l}0 . \\
68 \\
5\end{array}$ & $\begin{array}{l}- \\
0 . \\
23\end{array}$ & $\begin{array}{l}0 . \\
14 \\
7\end{array}$ & $\begin{array}{l}- \\
0 . \\
06\end{array}$ & $\begin{array}{l}0 . \\
23 \\
8\end{array}$ & $\begin{array}{l}0 . \\
1 \\
3\end{array}$ & $\begin{array}{l}0 . \\
03 \\
3\end{array}$ & $\begin{array}{l}- \\
0 . \\
08\end{array}$ & $\begin{array}{l}0 . \\
12 \\
1\end{array}$ & $\begin{array}{l}0 . \\
28 \\
2\end{array}$ & $\begin{array}{l}0 . \\
39 \\
3\end{array}$ & $\begin{array}{l}0 . \\
33 \\
8\end{array}$ & $\begin{array}{l}0 . \\
29 \\
6\end{array}$ & $\begin{array}{l}0 . \\
35 \\
7\end{array}$ & $\begin{array}{l}- \\
0 . \\
14 \\
3\end{array}$ & $\begin{array}{l}0 . \\
14 \\
9\end{array}$ & $\begin{array}{l}0 . \\
18\end{array}$ & $\begin{array}{l}0 . \\
05 \\
6\end{array}$ \\
\hline $\begin{array}{l}\text { Social } \\
\text { Media } \\
\text { Applicat } \\
\text { ion }\end{array}$ & $\begin{array}{l}0 . \\
66 \\
9\end{array}$ & $\begin{array}{l}0 . \\
13\end{array}$ & $\begin{array}{l}0 . \\
07 \\
1\end{array}$ & $\begin{array}{l}0 . \\
37 \\
8\end{array}$ & $\begin{array}{l}0 . \\
04 \\
7\end{array}$ & $\begin{array}{l}0 . \\
1\end{array}$ & $\begin{array}{l}0 . \\
00 \\
2\end{array}$ & $\begin{array}{l}- \\
0 . \\
26\end{array}$ & $\begin{array}{l}- \\
0 . \\
12\end{array}$ & $\begin{array}{l}0 . \\
39 \\
6\end{array}$ & $\begin{array}{l}0 . \\
43 \\
2\end{array}$ & $\begin{array}{l}0 . \\
31 \\
3\end{array}$ & $\begin{array}{l}0 . \\
20 \\
4\end{array}$ & $\begin{array}{l}- \\
0 . \\
04\end{array}$ & $\begin{array}{l}0 . \\
04 \\
7\end{array}$ & $\begin{array}{l} \\
0 . \\
3\end{array}$ & $\begin{array}{l}0 . \\
35 \\
7\end{array}$ & $\begin{array}{l}0 . \\
00 \\
6\end{array}$ \\
\hline Key Pad & $\begin{array}{l}0 . \\
55 \\
2\end{array}$ & $\begin{array}{l}0 . \\
02 \\
8\end{array}$ & $\begin{array}{l}0 . \\
20 \\
4\end{array}$ & $\begin{array}{l}0 . \\
05 \\
9\end{array}$ & $\begin{array}{l}0 . \\
34 \\
8\end{array}$ & $\begin{array}{l}0 . \\
1 \\
2\end{array}$ & $\begin{array}{l}- \\
0 . \\
55\end{array}$ & $\begin{array}{l}- \\
0 . \\
03\end{array}$ & $\begin{array}{l}0 . \\
03 \\
2\end{array}$ & $\begin{array}{l}0 . \\
06\end{array}$ & $\begin{array}{l}0 . \\
17\end{array}$ & $\begin{array}{l}0 . \\
28 \\
1\end{array}$ & $\begin{array}{l}0 . \\
12 \\
7\end{array}$ & $\begin{array}{l}0 . \\
40 \\
1\end{array}$ & $\begin{array}{l}0 . \\
00 \\
7\end{array}$ & $\begin{array}{l}. \\
01 \\
5\end{array}$ & $\begin{array}{l}0 . \\
70 \\
4\end{array}$ & $\begin{array}{l}- \\
0 . \\
05 \\
3\end{array}$ \\
\hline $\begin{array}{l}\text { Commu } \\
\text { nication }\end{array}$ & $\begin{array}{l}0 . \\
44 \\
3\end{array}$ & $\begin{array}{l}0 . \\
10 \\
3\end{array}$ & $\begin{array}{l}0 . \\
03 \\
5\end{array}$ & $\begin{array}{l}- \\
0 . \\
17\end{array}$ & $\begin{array}{l}0 . \\
50 \\
8\end{array}$ & $\begin{array}{l} \\
0 . \\
1\end{array}$ & $\begin{array}{l}- \\
0 . \\
27\end{array}$ & $\begin{array}{l}0 . \\
13 \\
3\end{array}$ & $\begin{array}{l}. \\
14 \\
6\end{array}$ & $\begin{array}{l}0 . \\
12 \\
5\end{array}$ & $\begin{array}{l}0 . \\
07 \\
9\end{array}$ & $\begin{array}{l}- \\
0 . \\
01 \\
7\end{array}$ & $\begin{array}{l}0 . \\
15 \\
3\end{array}$ & $\begin{array}{l}0 . \\
66\end{array}$ & $\begin{array}{l}0 . \\
05\end{array}$ & $\begin{array}{l}0 . \\
02 \\
1\end{array}$ & $\begin{array}{l}0 . \\
36 \\
7\end{array}$ & $\begin{array}{l}- \\
0 . \\
01 \\
3\end{array}$ \\
\hline $\begin{array}{l}\text { Sending } \\
\text { and } \\
\text { receivin } \\
\text { g e-mail }\end{array}$ & $\begin{array}{l}0 . \\
76 \\
1\end{array}$ & $\begin{array}{l}0 . \\
2\end{array}$ & $\begin{array}{l}- \\
0 . \\
36\end{array}$ & $\begin{array}{l}0 . \\
06 \\
7\end{array}$ & $\begin{array}{l}- \\
0 . \\
16\end{array}$ & -0 & $\begin{array}{l} \\
0 . \\
07\end{array}$ & $\begin{array}{l}- \\
0 . \\
02\end{array}$ & $\begin{array}{l}- \\
0 . \\
1 \\
6\end{array}$ & $\begin{array}{l}0 . \\
70 \\
4\end{array}$ & $\begin{array}{l}0 . \\
26 \\
7\end{array}$ & $\begin{array}{l}0 . \\
17 \\
5\end{array}$ & $\begin{array}{l}- \\
0 . \\
04\end{array}$ & $\begin{array}{l}0 . \\
05 \\
7\end{array}$ & $\begin{array}{l}0 . \\
38 \\
2\end{array}$ & $\begin{array}{l} \\
0 . \\
01\end{array}$ & $\begin{array}{l}0 . \\
25\end{array}$ & $\begin{array}{l}- \\
0 . \\
00 \\
4\end{array}$ \\
\hline
\end{tabular}




\begin{tabular}{|c|c|c|c|c|c|c|c|c|c|c|c|c|c|c|c|c|c|c|}
\hline $\begin{array}{l}\text { As a } \\
\text { modem }\end{array}$ & $\begin{array}{l}0 . \\
45 \\
5\end{array}$ & $\begin{array}{l}0 . \\
31 \\
9\end{array}$ & $\begin{array}{l}0 . \\
11 \\
6\end{array}$ & $\begin{array}{l}0 . \\
07 \\
8\end{array}$ & $\begin{array}{l}0 . \\
10 \\
5\end{array}$ & $\begin{array}{l}0 . \\
3 \\
3\end{array}$ & $\begin{array}{l}- \\
0 . \\
42\end{array}$ & $\begin{array}{l}0 . \\
08 \\
6\end{array}$ & $\begin{array}{l}-\overline{0} \\
0 . \\
36\end{array}$ & $\begin{array}{l}0 . \\
23 \\
6\end{array}$ & $\begin{array}{l}0 . \\
03 \\
6\end{array}$ & $\begin{array}{l}0 . \\
04 \\
9\end{array}$ & $\begin{array}{l}0 . \\
15 \\
9\end{array}$ & $\begin{array}{l}-\overline{ } \\
0 . \\
04\end{array}$ & $\begin{array}{l}0 . \\
18 \\
2\end{array}$ & $\begin{array}{l}0 . \\
01 \\
3\end{array}$ & $\begin{array}{l}0 . \\
77 \\
9\end{array}$ & $\begin{array}{l}0 . \\
17 \\
5\end{array}$ \\
\hline $\begin{array}{l}\text { Playing } \\
\text { games }\end{array}$ & $\begin{array}{l}0 . \\
48 \\
2\end{array}$ & $\begin{array}{l}0 . \\
10 \\
8\end{array}$ & $\begin{array}{l}0 . \\
58 \\
7\end{array}$ & $\begin{array}{l}- \\
0 . \\
13\end{array}$ & $\begin{array}{l}- \\
0 . \\
18\end{array}$ & $\begin{array}{l}- \\
0 . \\
2\end{array}$ & $\begin{array}{l}- \\
0 . \\
05\end{array}$ & $\begin{array}{l}- \\
0 . \\
14\end{array}$ & $\begin{array}{l}0 . \\
20 \\
9\end{array}$ & $\begin{array}{l}- \\
0 . \\
03 \\
2 \\
\end{array}$ & $\begin{array}{l}0 . \\
06 \\
1\end{array}$ & $\begin{array}{l}0 . \\
46 \\
3\end{array}$ & $\begin{array}{l}0 . \\
61 \\
8\end{array}$ & $\begin{array}{l}0 . \\
13 \\
2\end{array}$ & $\begin{array}{l}0 . \\
23 \\
9\end{array}$ & $\begin{array}{l}0 . \\
01\end{array}$ & $\begin{array}{l}0 . \\
07 \\
5\end{array}$ & $\begin{array}{l}- \\
0 . \\
22 \\
3\end{array}$ \\
\hline $\begin{array}{l}\text { Strong } \\
\text { battery } \\
\text { back-up }\end{array}$ & $\begin{array}{l}0 . \\
69 \\
6 \\
\end{array}$ & $\begin{array}{l}- \\
0 . \\
19\end{array}$ & $\begin{array}{l}- \\
0 . \\
06\end{array}$ & $\begin{array}{l}- \\
0 . \\
23\end{array}$ & $\begin{array}{l}- \\
0 . \\
15\end{array}$ & $\begin{array}{l}0 . \\
1 \\
6 \\
\end{array}$ & $\begin{array}{l}0 . \\
02\end{array}$ & $\begin{array}{l}0 . \\
17 \\
6 \\
\end{array}$ & $\begin{array}{l}0 . \\
13 \\
3 \\
\end{array}$ & $\begin{array}{l}0 . \\
43 \\
2 \\
\end{array}$ & $\begin{array}{l}0 . \\
17 \\
8\end{array}$ & $\begin{array}{l}0 . \\
40 \\
5 \\
\end{array}$ & $\begin{array}{l}0 . \\
15 \\
1 \\
\end{array}$ & $\begin{array}{l}0 . \\
24 \\
9 \\
\end{array}$ & $\begin{array}{l}0 . \\
15 \\
9 \\
\end{array}$ & $\begin{array}{l}0 . \\
38 \\
9 \\
\end{array}$ & $\begin{array}{l}0 . \\
02 \\
9 \\
\end{array}$ & $\begin{array}{l}0 . \\
16 \\
5\end{array}$ \\
\hline $\begin{array}{l}\text { Design } \\
\& \\
\text { Appeara } \\
\text { nce }\end{array}$ & $\begin{array}{l}0 . \\
56 \\
1\end{array}$ & $\begin{array}{l}- \\
0 . \\
1\end{array}$ & $\begin{array}{l}-\overline{0} \\
0 . \\
22\end{array}$ & $\begin{array}{l}- \\
0 . \\
17\end{array}$ & $\begin{array}{l}0 . \\
33 \\
8\end{array}$ & $\begin{array}{l}- \\
0 . \\
3\end{array}$ & $\begin{array}{l}0 . \\
04 \\
5\end{array}$ & $\begin{array}{l}0 . \\
23 \\
5\end{array}$ & $\begin{array}{l}0 . \\
36 \\
7\end{array}$ & $\begin{array}{l}0 . \\
29 \\
4\end{array}$ & $\begin{array}{l}0 . \\
22 \\
9\end{array}$ & $\begin{array}{l}0 . \\
09 \\
9\end{array}$ & $\begin{array}{l}0 . \\
01 \\
4\end{array}$ & $\begin{array}{l}0 . \\
78\end{array}$ & $\begin{array}{l}0 . \\
16 \\
1\end{array}$ & $\begin{array}{l}0 . \\
05 \\
6\end{array}$ & $\begin{array}{l}- \\
0 . \\
13\end{array}$ & $\begin{array}{l}0 . \\
02 \\
6\end{array}$ \\
\hline $\begin{array}{l}\text { Sophisti } \\
\text { cated }\end{array}$ & $\begin{array}{l}0 . \\
54 \\
4\end{array}$ & $\begin{array}{l}0 . \\
32 \\
2\end{array}$ & $\begin{array}{l}- \\
0 . \\
26\end{array}$ & $\begin{array}{l}0 . \\
12 \\
5\end{array}$ & $\begin{array}{l}- \\
0 . \\
07\end{array}$ & $\begin{array}{l}- \\
0 . \\
4\end{array}$ & $\begin{array}{l}- \\
0 . \\
09\end{array}$ & $\begin{array}{l}0 . \\
08 \\
8\end{array}$ & $\begin{array}{l}- \\
0 . \\
22\end{array}$ & $\begin{array}{l}0 . \\
42\end{array}$ & $\begin{array}{l}0 . \\
26\end{array}$ & $\begin{array}{l}- \\
0 . \\
05 \\
9\end{array}$ & $\begin{array}{l}-\overline{0} \\
03\end{array}$ & $\begin{array}{l}0 . \\
12 \\
8\end{array}$ & $\begin{array}{l}0 . \\
59\end{array}$ & $\begin{array}{l}- \\
0 . \\
19\end{array}$ & $\begin{array}{l}0 . \\
18\end{array}$ & $\begin{array}{l}- \\
0 . \\
08 \\
8\end{array}$ \\
\hline $\begin{array}{l}\text { Good } \\
\text { audio- } \\
\text { video } \\
\text { quality }\end{array}$ & $\begin{array}{l}0 . \\
66 \\
2\end{array}$ & $\begin{array}{l}0 . \\
23 \\
7\end{array}$ & $\begin{array}{l}0 . \\
00 \\
1\end{array}$ & $\begin{array}{l}- \\
0 . \\
25\end{array}$ & $\begin{array}{l}- \\
0 . \\
15\end{array}$ & $\begin{array}{l}- \\
0 . \\
2\end{array}$ & $\begin{array}{l}- \\
0 . \\
14\end{array}$ & $\begin{array}{l}0 . \\
21 \\
6\end{array}$ & $\begin{array}{l}0 . \\
05 \\
6\end{array}$ & $\begin{array}{l}0 . \\
37 \\
7\end{array}$ & $\begin{array}{l}0 . \\
02 \\
3\end{array}$ & $\begin{array}{l}0 . \\
19 \\
7\end{array}$ & $\begin{array}{l}0 . \\
25\end{array}$ & $\begin{array}{l}0 . \\
31 \\
9\end{array}$ & $\begin{array}{l}0 . \\
53 \\
8\end{array}$ & $\begin{array}{l}0 . \\
15 \\
3\end{array}$ & $\begin{array}{l}0 . \\
15\end{array}$ & $\begin{array}{l}- \\
0 . \\
02 \\
8\end{array}$ \\
\hline $\begin{array}{l}\text { Operatin } \\
\text { g } \\
\text { System }\end{array}$ & $\begin{array}{l}0 . \\
70 \\
4\end{array}$ & $\begin{array}{l}0 . \\
15 \\
9\end{array}$ & $\begin{array}{l}- \\
0 . \\
34\end{array}$ & $\begin{array}{l}- \\
0 . \\
11\end{array}$ & $\begin{array}{l}0 . \\
26 \\
5\end{array}$ & $\begin{array}{l}0 . \\
0 \\
2\end{array}$ & $\begin{array}{l}0 . \\
20 \\
8\end{array}$ & $\begin{array}{l}- \\
0 . \\
1\end{array}$ & -0 & $\begin{array}{l}0 . \\
73 \\
4\end{array}$ & $\begin{array}{l}0 . \\
28 \\
9\end{array}$ & $\begin{array}{l}- \\
0 . \\
03 \\
2\end{array}$ & $\begin{array}{l}0 . \\
14 \\
6\end{array}$ & $\begin{array}{l}0 . \\
34 \\
4\end{array}$ & $\begin{array}{l}0 . \\
01 \\
6\end{array}$ & $\begin{array}{l}- \\
0 . \\
05\end{array}$ & $\begin{array}{l}0 . \\
08 \\
7\end{array}$ & $\begin{array}{l}0 . \\
01 \\
7\end{array}$ \\
\hline $\begin{array}{l}\text { Video- } \\
\text { calling } \\
\text { facility }\end{array}$ & $\begin{array}{l}0 . \\
66 \\
8\end{array}$ & $\begin{array}{l}0 . \\
39\end{array}$ & $\begin{array}{l}- \\
0 . \\
32\end{array}$ & $\begin{array}{l}- \\
0 . \\
18\end{array}$ & $\begin{array}{l}- \\
0 . \\
13\end{array}$ & $\begin{array}{l}0 . \\
0 \\
9\end{array}$ & $\begin{array}{l}0 . \\
14\end{array}$ & $\begin{array}{l}- \\
0 . \\
01\end{array}$ & $\begin{array}{l}0 . \\
17 \\
5\end{array}$ & $\begin{array}{l}0 . \\
79 \\
4\end{array}$ & $\begin{array}{l}- \\
0 . \\
08 \\
2\end{array}$ & $\begin{array}{l}0 . \\
18 \\
5\end{array}$ & $\begin{array}{l}0 . \\
16 \\
8\end{array}$ & $\begin{array}{l}0 . \\
20 \\
6\end{array}$ & $\begin{array}{l}0 . \\
24 \\
3\end{array}$ & $\begin{array}{l}- \\
0 . \\
04\end{array}$ & $\begin{array}{l}0 . \\
01 \\
6\end{array}$ & $\begin{array}{l}0 . \\
03 \\
2\end{array}$ \\
\hline $\begin{array}{l}\text { Multi- } \\
\text { Window }\end{array}$ & $\begin{array}{l}0 . \\
66 \\
7 \\
\end{array}$ & $\begin{array}{l}0 . \\
28 \\
2\end{array}$ & $\begin{array}{l}- \\
0 . \\
39\end{array}$ & $\begin{array}{l}0 . \\
16 \\
4\end{array}$ & $\begin{array}{l}- \\
0 . \\
18\end{array}$ & $\begin{array}{l}0 . \\
1 \\
5\end{array}$ & $\begin{array}{l}- \\
0 . \\
03\end{array}$ & $\begin{array}{l}- \\
0 . \\
08\end{array}$ & $\begin{array}{l}0 . \\
09 \\
7\end{array}$ & $\begin{array}{l}0 . \\
73 \\
7\end{array}$ & $\begin{array}{l}0 . \\
07 \\
3\end{array}$ & $\begin{array}{l}0 . \\
32 \\
4 \\
\end{array}$ & $\begin{array}{l}- \\
0 . \\
09\end{array}$ & $\begin{array}{l}0 . \\
05 \\
5 \\
\end{array}$ & $\begin{array}{l}0 . \\
21 \\
8\end{array}$ & $\begin{array}{l}- \\
0 . \\
17\end{array}$ & $\begin{array}{l}0 . \\
18 \\
1 \\
\end{array}$ & $\begin{array}{l}0 . \\
03 \\
8\end{array}$ \\
\hline
\end{tabular}

Source: Primary (Data processed through SPSS 16.0), Extraction Method: Principal Component Analysis, Rotation Method: Varimax with Kaiser Normalization. Rotation converged in 19 iterations.

Table IV shows the component matrix or factor matrix. It is also called factor pattern matrix. It shows the coefficient used to express the standardized variables in the terms of the factors. These coefficients, the factors loadings, represent the correlation between the factors and variables. A coefficient with a large absolute value indicates that the factors and the variable are closely related. The left portion of the table namely component matrix indicates the relationship between the factors and individual variables; it seldom result in the factors that can be interpreted, because factors are correlated with many variables. Therefore right portion of the table 4 namely rotated component matrixes is useful for interpreting the factors. The rotation is made by Varimax procedure. This is an orthogonal (unrelated) method of rotation that minimizes the number of variables with high loading on a factor, thereby enhancing the interpretability of the factors. Interpretation of facilitated by identifying the variables that have large loadings on the same factors, the factor can be interpreted in terms of the variables that load high on it. For the purpose of interpretation, each factor was composed of variable that loaded 0.30 or higher on that factor. In case a variables loaded 0.30 or above on two factors, each variable was assigned to factor where it had the highest loading. The maximum of each row (ignoring the sign) indicates the respective variable belongs to the respective component (Table IV). 
IOSR Journal of Business and Management (IOSR-JBM)

e-ISSN: 2278-487X, p-ISSN: 2319-7668

TABLE V: Component Score Coefficient Matrix

\begin{tabular}{|c|c|c|c|c|c|c|c|c|c|}
\hline & 1 & 2 & 3 & 4 & 5 & 6 & 7 & 8 & 9 \\
\hline Camera & .044 & -.081 & .237 & .041 & -.137 & -.111 & .340 & -.016 & -.027 \\
\hline Bluetooth & -.007 & -.089 & -.069 & .406 & .038 & -.043 & -.135 & -.057 & .113 \\
\hline \begin{tabular}{|ll} 
MP & 3 \\
player & \\
\end{tabular} & -.039 & .118 & -.167 & .358 & -.113 & .029 & .035 & .065 & -.090 \\
\hline Dual Sim & -.051 & -.007 & .295 & .016 & -.058 & .050 & -.117 & -.143 & .252 \\
\hline Wi-Fi & -.090 & .044 & .308 & -.078 & .001 & -.059 & -.049 & .067 & .032 \\
\hline FM-Radio & .085 & -.012 & .125 & .138 & -.053 & -.146 & -.483 & -.102 & -.022 \\
\hline $\begin{array}{l}\text { Brand } \\
\text { image }\end{array}$ & .006 & -.097 & .371 & -.121 & -.010 & -.011 & .011 & .018 & -.195 \\
\hline Shape & -.047 & .264 & -.043 & -.020 & .129 & -.047 & -.053 & -.061 & -.076 \\
\hline \begin{tabular}{|l} 
Size of \\
mobile \\
phone
\end{tabular} & -.017 & .308 & -.051 & -.055 & -.062 & -.126 & .007 & .079 & .030 \\
\hline Weight & -.031 & -.025 & -.050 & -.027 & .013 & .031 & .048 & .050 & .769 \\
\hline Colour & -.170 & .336 & -.008 & -.118 & .036 & .264 & -.109 & -.155 & .023 \\
\hline $\begin{array}{l}\text { Video- } \\
\text { recorder }\end{array}$ & -.088 & -.066 & .014 & .080 & -.071 & .474 & .101 & -.049 & .092 \\
\hline \begin{tabular}{|l|} 
Touch \\
Screen \\
\end{tabular} & -.005 & .321 & -.201 & .125 & -.234 & .080 & .175 & .001 & -.047 \\
\hline $\begin{array}{l}\text { Internet/G } \\
\text { PRS }\end{array}$ & .102 & .079 & -.059 & .187 & .059 & -.162 & .115 & -.178 & .144 \\
\hline $\begin{array}{l}\text { Huge } \\
\text { Memory }\end{array}$ & .310 & .025 & -.099 & .012 & -.143 & -.162 & .103 & -.027 & -.050 \\
\hline $\begin{array}{l}\text { Screen } \\
\text { size }\end{array}$ & .003 & .052 & .081 & .082 & .134 & -.214 & .030 & .023 & .012 \\
\hline \begin{tabular}{|l|} 
Social \\
Media \\
Applicatio \\
$\mathrm{n}$
\end{tabular} & .074 & .136 & .037 & .037 & -.168 & -.108 & -.188 & .154 & -.067 \\
\hline Key Pad & -.136 & -.052 & .081 & -.061 & .198 & -.080 & .006 & .456 & -.080 \\
\hline \begin{tabular}{|l|}
$\begin{array}{l}\text { Communi } \\
\text { cation }\end{array}$ \\
\end{tabular} & -.090 & -.068 & -.076 & -.010 & .415 & -.020 & -.011 & .193 & -.012 \\
\hline $\begin{array}{l}\text { Sending } \\
\text { and } \\
\text { receiving } \\
\text { e-mail }\end{array}$ & .178 & .024 & -.055 & -.116 & -.115 & .142 & -.007 & .086 & -.028 \\
\hline $\begin{array}{|ll|}\text { As } & \mathrm{a} \\
\text { modem }\end{array}$ & -.004 & -.048 & -.103 & -.018 & -.150 & .053 & .074 & .544 & .127 \\
\hline \begin{tabular}{|l|}
$\begin{array}{l}\text { Playing } \\
\text { games }\end{array}$ \\
\end{tabular} & -.131 & -.085 & .224 & .249 & .045 & .070 & -.037 & -.058 & -.207 \\
\hline \begin{tabular}{|l} 
Strong \\
battery \\
back-up
\end{tabular} & .062 & -.095 & .141 & -.010 & .069 & .015 & .193 & -.078 & .140 \\
\hline
\end{tabular}


IOSR Journal of Business and Management (IOSR-JBM) e-ISSN: 2278-487X, p-ISSN: 2319-7668

\begin{tabular}{|l|l|l|l|l|l|l|l|l|l|}
\hline $\begin{array}{l}\text { Design \& } \\
\text { Appearan } \\
\text { ce }\end{array}$ & -.028 & -.028 & -.005 & -.068 & .513 & .056 & -.037 & -.213 & .027 \\
\hline $\begin{array}{l}\text { Sophistica } \\
\text { ted }\end{array}$ & .042 & .102 & -.187 & -.094 & -.012 & .350 & -.101 & .051 & -.085 \\
\hline $\begin{array}{l}\text { Good } \\
\text { audio- } \\
\text { video } \\
\text { quality }\end{array}$ & -.006 & -.129 & .009 & .019 & .135 & .291 & .080 & -.001 & -.009 \\
\hline $\begin{array}{l}\text { Operating } \\
\text { System }\end{array}$ & .233 & .050 & -.160 & .028 & .097 & -.126 & -.072 & -.057 & -.010 \\
\hline $\begin{array}{l}\text { Video- } \\
\text { calling } \\
\text { facility }\end{array}$ & .267 & -.196 & .041 & .019 & .041 & .017 & -.049 & -.121 & .019 \\
\hline $\begin{array}{l}\text { Multi- } \\
\text { Window }\end{array}$ & .235 & -.113 & .117 & -.129 & -.068 & .005 & -.109 & .024 & -.005 \\
\hline
\end{tabular}

Extraction Method: Principal Component Analysis.

Rotation Method: Varimax with Kaiser Normalization.

Source: Primary (Data processed through SPSS 16.0)

Extraction Method: Principal Component Analysis.

Table VI defined the light on attributes which are considered by consumers before making purchase decisions. Factor i.e. Technical Features are most considered attributes of mobile phones as it accounts for $32.479 \%$ of the total variance explained, followed by Looks, image with Resource,Entertainment, Basic Attributes, Storage \& discussion way entertainment, User Friendly and Weight.

TABLE VI: Attributes Influencing the Consumer for Purchase of Mobile Phones

\begin{tabular}{|l|l|l|l|l|}
\hline Factor No. & Name of Dimension & Variable & $\begin{array}{l}\text { Variables } \\
\text { No. }\end{array}$ & Factor loading \\
\hline F1 & Technical Features & Internet/GPRS & 14 & .432 \\
& $(32.479 \%)$ & Huge Memory & 15 & .794 \\
& & Sending \& receiving e-mail & 20 & .704 \\
& & Strong battery back-up & 23 & .432 \\
& & Operating System & 27 & .734 \\
& & Video-calling Facility & 28 & .794 \\
\hline F2 & Multi- Window & 29 & .737 \\
& Looks & Shape & 8 & .678 \\
& $(7.159 \%)$ & Size & 9 & .720 \\
& & Colour & 11 & .720 \\
& & Touch-Screen & 13 & .642 \\
& & Screen size & 16 & .393 \\
& & Social Media Application & 17 & .432 \\
\hline F3 & Image with Resource & Dual Sim & 4 & .604 \\
& $(6.822 \%)$ & Wi-fi & 5 & .664 \\
& & Brand Image & 7 & .674 \\
\hline F5 & Entertainment & Bluetooth & 2 & .790 \\
& $(6.268 \%)$ & MP 3 Player & 3 & .764 \\
& & Playing Games & 22 & .463 \\
\hline
\end{tabular}


IOSR Journal of Business and Management (IOSR-JBM)

\begin{tabular}{|l|l|l|l|l|}
\hline F6 & $\begin{array}{l}\text { Storage \& Display } \\
(4.253 \%)\end{array}$ & $\begin{array}{l}\text { Video-recorder } \\
\text { Sophisticated } \\
\text { Good audio-video quality }\end{array}$ & 12 & .737 \\
& & Camera & 1 & .590 \\
\hline F7 & Way Entertainment & FM-Radio & 6 & .638 \\
\hline F8 & User Friendly & Key Pad & 18 & .809 \\
& $(3.730 \%)$ & As a modem & 21 & .704 \\
\hline
\end{tabular}

\section{CONCLUSION}

In total, it is found that Technical Features are most considered attributes of mobile phone followed by Looks, Image \& Resource, Entertainment, Basic Attributes, Storage, Display, Way Entertainment, User Friendly and Weight. Technical attributes includes Internet/GPRS, Huge Memory, Sending \& receiving e-mail, Strong battery back-up, Operating System, Video-calling Facility and Multi- Window. Looks encompasses Shape, Size, Colour, Touch-Screen, Screen size and Social Media Application. Image with Resource involves Dual Sim, Wi-fi and Brand Image. Entertainment attributes incorporates Bluetooth, MP 3 Player and Playing Games. Basic Attributes includes Communication and Design \& Appearance. Storage \& Display attributes contains Video-recorder, Sophisticated and Good audio-video quality. Way Entertainment attributes embraces Camera and FM-Radio. User friendly attributes includes Key Pad and as a modem.

\section{REFERENCES}

[1] S. Mokhlis and A. Y. Yakoop, "Consumer Choice criteria in Mobile Phone selection: An investigation of Malaysian University student”, International review of Social Science \& Humanities, 2(2), 2012, 203-212.

[2] Macro Analysis \& Consumer Research Organisation (2004). Study of Mobile Phone Usage Among the Teenagers And Youth In Mumbai. Reterived from, https://www.itu.int/osg/spu/ni/futuremobile/socialaspects/IndiaMacroMobileYouthStudy04.pdf.

[3] M Chowdhury and M. T. Rahman, "Consumer Attitude Towards the Cell Phone; A Study on Young Generations of Chittagong Metropolitan city, Bangladesh", Asian Business Review, 3(5), 2013, 16-20.

[4] P Gopal, Anjali and Aakanksha, "Mobile Marketing Research: A Study of Brand Prefrence by VIT Student's", International Journal of Advanced Research in Computer Science and Software Engineering, 11, 2013, 1620-1623.

[5] S. Malviya, M. S. Saluja and A. S. Thakur, "A Study of the Factors Influencing Consumer's Purchase Decision towards Smartphones in Indore". International Journal of Advanced Research in Computer Science and Management Studies, 1(6), 2013, 14-21.

[6] R. Rijial, "Brand preference on Mobile Purchase among the Students of Roskilde University", Bechelor of Business studies Project report, Roskilde University.

[7] M. Sata, "Factors Affecting Consumer Buying Behaviour of Mobile Phone", Mediterranean Journal of Social Science, 4 (12), 2013, 103-112.

[8] K. G. Singh, "Customer Preferences and Satisfaction towards Mobile Phone- The Case of Selected District of Punjab", Asian Journal of Research Marketing, 1(4), 2012, 16-31.

[9] J. Marumba, "A Pragmatic Assesment of the Determinants of Consumer Brand Prefernce in the Mobile Telecommunication Services Market in Masvingo Urban" Zimbabwe, Management and Administrative Science Review, 2(2), 2013, 144-155. 